\title{
Clinical Outcomes and Therapeutic Strategy in Patients With Acute Myocardial Infarction According to Renal Function
}

\author{
Data From the Korean Acute Myocardial \\ Infarction Registry
}

\author{
Sang-Hee Lee, MD ${ }^{1}$; Young-Jo Kim, MD ${ }^{1}$; Woong Kim, MD ${ }^{1}$; Jong-Seon Park, MD ${ }^{1}$; \\ Dong-Gu Shin, MD ${ }^{1}$; Seung-Ho Hur, MD²; Chong-Jin Kim, MD³; Myeong-Chan Cho, MD; \\ Shung-Chull Chae, MD ${ }^{5}$; Myung-Ho Jeong, MD ${ }^{6}$; Taek-Jong Hong, $\mathrm{MD}^{7}$; \\ Doo-Il Kim, MD ${ }^{8}$; Kee-Sik Kim, MD $^{9}$ and other \\ Korean Acute Myocardial Infarction Registry Investigators ${ }^{10}$
}

\begin{abstract}
Background The aim of the present study was to evaluate the relationship between clinical outcomes after acute myocardial infarction (MI) and renal function by glomerular filtration rate (GFR) in patients with normal or mildly elevated serum creatinine concentrations.

Methods and Results As part of the Korean Acute Myocardial Infarction Registry (KAMIR), 6,834 acute MI patients with a serum creatinine concentration of $\leq 2.0 \mathrm{mg} / \mathrm{dl}$ were enrolled from November 2005 to December 2006. The renal function was stratified arbitrary to 5 groups: (1) normal function, $>90.0$; (2) preserved function, 75.0-89.9; (3) mild dysfunction, 60.0-74.9; (4) moderate dysfunction, 45.0-59.9; (5) severe dysfunction, $<45 \mathrm{ml} \cdot \mathrm{min}^{-1} \cdot 1.73 \mathrm{~m}^{-2}$. Clinical characteristics, mortality and adverse events were analyzed among each group. Although reperfusion and medical therapies were underused, the rates of mortality and adverse events were increased with declining renal function. After adjustment with confounders, severe and moderate renal dysfunctions were important risk predictors of in-hospital mortality, long-term mortality and adverse events.

Conclusion The spectrum of renal function, when it was presented by GFR, is broad and is an important risk predictor for adverse outcomes after acute MI, even in patients with normal or mildly elevated serum creatinine concentrations. Furthermore, standard treatments were underused in any degree of renal dysfunction. (Circ J 2008; 72: $1410-1418$ )
\end{abstract}

Key Words: Glomerular filtration rate; Mortality; Myocardial infarction

$\mathbf{T}$ he renal dysfunction has been reported as an independent risk factor which has shown worse clinical outcomes after acute myocardial infarction (MI) ${ }^{1-3}$ Previous studies, which have focused on patients with severe and end-stage renal disease, showed that high inci-

(Received January 29, 2008; revised manuscript received March 31, 2008; accepted April 24, 2008)

${ }^{1}$ Division of Cardiology, Yeungnam University Hospital, ${ }^{2}$ Division of Cardiology, Keimyung University Dongsan Hospital, Daegu, ${ }^{3}$ Division of Cardiology, Kyunghee University Hospital, Seoul, ${ }^{4}$ Division of Cardiology, Chungbuk National University Hospital, Chungju, ${ }^{5}$ Division of Cardiology, Kyungpook National University Hospital, Daegu, ${ }^{6}$ Division of Cardiology, Chonnam National University Hospital, Gwangju, ${ }^{7}$ Division of Cardiology, Pusan University Hospital, ${ }^{8}$ Division of Cardiology, Inje University Baek Hospital, Pusan, ${ }^{9}$ Division of Cardiology, Daegu Catholic University Hospital, Daegu, Republic of Korea

${ }^{10}$ Korea Acute Myocardial Infarction Registry (KAMIR) Study Group of Korean Circulation Society members are listed in Appendix 1.

Mailing address: Young-Jo Kim, MD, Division of Cardiology, Yeungnam University Hospital, 5 Daemyoung Dong, Nam-Gu, Daegu 705-717, Republic of Korea. E-mail: yjkim@med.yu.ac.kr All rights are reserved to the Japanese Circulation Society. For permissions, please e-mail: cj@j-circ.or.jp dence of mortality and adverse events is observed in those patients who developed acute MI4,5 This particularly poor outcome has been explained by facts such as specific pathophysiologic condition, high prevalence of comorbidity and underuse of life-saving treatments, so called 'therapeutic nihilism'6 However, the relationship between clinical outcomes and the utilization of standard therapies after acute MI and renal function is not clear yet in patients with normal or mildly elevated serum creatinine concentrations, because those patients have not been highlighted in studies of this area. In fact, serum creatinine concentration has been known as an insensitive indicator of renal function! Despite a relatively normal range of serum creatinine concentration, some patients might have renal dysfunction or impairment, when renal function is defined by estimated glomerular filtration rate (GFR). Furthermore, any degree of renal dysfunction, as well as end-stage renal disease, might be an indicator of early pathologic changes of vascular wall such as atherosclerosis, and could be associated with poor prognosis after acute MI ${ }^{7}, 8$

Consequently, as part of the Korean Acute Myocardial Infarction Registry (KAMIR) study, the present study aimed to investigate the prognostic impact of renal function 
by estimated GFR, and test the effects of evidence-based therapies in unselected large populations with normal or mildly elevated serum creatinine concentration who developed acute MI, to coincide with real practice.

\section{Methods}

\section{Patients and Baseline Characteristics}

The KAMIR study is a retrospective multi-hospital study and collected data from 10,463 patients with acute MI between November 2005 and December 2006. The purpose and methods used to register patients of the KAMIR study have been described previously?

We abstracted 7,411 data that were available to calculate the estimated GFR. Of these patients, we analyzed data of 6,834 eligible patients who were 20 years or older (mean age: $64.1 \pm 12.9$ years, male: $n=5,209)$. These patients were newly diagnosed with acute $\mathrm{MI}$ at 41 primary percutaneous coronary intervention (PCI) facilities and admitted to hospital within 7 days after symptom onset. On the basis of the cut-off value of serum creatinine which was used in the Third National Health and Nutrition Examination Survey (NHANES III) ${ }^{10}$ patients with a baseline serum creatinine concentration of more than $2.0 \mathrm{mg} / \mathrm{dl}$, as the index of moderate and severe renal impairment, were excluded. We also excluded patients with a history of previous renal diseases and overt renal failure (defined as renal replacement therapy or GFR of less than $15 \mathrm{ml}$ per min per $1.73 \mathrm{~m}^{2}$ of body surface area). The clinical data after discharge were obtained from outpatient visits and contacted by telephone at 30, 90 and 180 days.

\section{Definition}

Acute MI was defined by World Health Organization criteria incorporating clinical features, elevated biochemistry marker and electrocardiographic findings ${ }^{11}$ ST elevation MI (STEMI) was indicated by new ST elevation in at least 2 contiguous leads, measuring more than $0.2 \mathrm{mV}$ in leads $\mathrm{V}_{1-3}$, or $0.1 \mathrm{mV}$ in all other leads. When these findings were compounded, the diagnosis was supported by coronary angiographic (CAG) findings. Killip class was defined at admission. Left ventricular ejection fraction (LVEF) was shown by echocardiography.

\section{GFR Measurement}

Renal function was defined by estimated GFR. Of several reliable equations, we used the Mayo clinic quadratic equation (MCQ) because of the following reasons: (1) Cockcroft and Gault formula usually overestimates GFR, especially in low range; and (2) the modification of diet in renal disease equation underestimates high GFR in diabetics. ${ }^{2}$ MCQ incorporated age, sex and serum creatinine concentration.

$$
\begin{aligned}
\mathrm{MCQ}= & \exp \left[1.911+5.249 / \mathrm{SCr}-2.114 / \mathrm{SCr}^{2}-0.00686 \times\right. \\
& \text { age (years) }-0.205 \text { if female }] .
\end{aligned}
$$

According to the cut-off value which was used in the Candesartan in heart failure: Assessment of Reduction in Mortality and Morbidity (CHARM) trial, ${ }^{13}$ we stratified a study population into 5 groups as follows: normal renal function, >90.0; preserved renal function, 75.0-89.9; mild renal dysfunction, 60.0-74.9; moderate renal dysfunction, 45.059.9; severe renal dysfunction, $<45 \mathrm{ml} \cdot \mathrm{min}^{-1} \cdot 1.73 \mathrm{~m}^{-2}$. Serum creatinine concentration used in this equation was an initial value after presentation and had a normal range from $0.6-1.5 \mathrm{mg} / \mathrm{dl}$.

\section{Treatment}

PCI and coronary artery bypass graft (CABG) surgery were used for reperfusion therapy. As primary reperfusion therapy in STEMI patients, intravenous thrombolytic therapy, primary and facilitated PCI were performed. Early invasive PCI was defined as emergent PCI, which was performed in non-STEMI (NSTEMI) patients with remained symptoms. Treatment strategies were decided by operators, according to the American College of Cardiology and the American Heart Association guidelines for the management of patients with acute coronary syndrome. Furthermore, evidence-based medications were prescribed in a non-randomized unselected condition.

\section{Clinical Outcomes}

The primary endpoint was death from any cause. Other major adverse cardiac events such as cardiac death, recurrent MI, repeated coronary revascularization and CABG were defined as secondary endpoints. Cardiac death included sudden death within 1 month after MI onset as well as a known mechanical or arrhythmic cause.

\section{Statistical Analysis}

The estimated GFR is presented in categorical value which was suited for our purposes. For continuous variables, comparison among each group was performed by oneway ANOVA and the Kruskal-Wallis test, when appropriate. Pearson's chi-square test was used for the analysis of categorical variables. All p-values were two-sided, and a p-value of less than 0.05 was considered to indicate statistical significance. Univariate and multivariate analyses were performed to determine the prognostic significance of clinical variables for in-hospital and long-term clinical endpoint. Variables with p-values of $<0.25$ on univariate analysis were entered into multivariate logistic regression models and Cox proportional-hazards models. Multivariate logistic regression modeling was used to compare in-hospital outcomes and candidates for adjustment included age, sex, comorbidities (previous MI, previous PCI, diabetes, hypertension, elevated lipid concentrations, current smoking, previous stroke, previous heart failure, chronic lung disease), 'symptom to door time', Killip class $>$ I, primary ventricular tachyarrhythmia, LVEF $<40 \%$, therapeutic modalities and medical treatments during hospitalization, angiographic findings, PCI-related complications, and spectrum of renal function. Cox proportional-hazards modeling was used to compare long-term clinical outcomes. The following variables were included in adjustment: age, sex, comorbidities (previous MI, previous PCI, diabetes, hypertension, elevated lipid concentraitons, current smoking, previous stroke, previous heart failure, peripheral vascular disease, malignancy), pre-hospital resuscitation, 'symptom to door time', symptoms and sign at presentation, Killip class $>$ I, atrial fibrillation, primary ventricular tachyarrhythmia, LVEF $<40 \%$, therapeutic modalities, medical treatments during hospitalization and at discharge, angiographic findings, PCI-related complications, and spectrum of renal function. We used stepwise elimination and backward selections to select the most powerful predictive variables in both models. Life table estimates, by 5 groups according to the estimated GFR, for long-term mortality and adverse events were determined and presented as event curves. Analyses were performed with the use of SPSS software (version 13.0, SPSS Inc, Chicago, IL, USA). 
Table 1 Baseline Characteristics According to the eGFR

\begin{tabular}{|c|c|c|c|c|c|c|}
\hline & $\begin{array}{l}\text { Normal renal } \\
\text { function }\end{array}$ & $\begin{array}{l}\text { Preserved renal } \\
\quad \text { function }\end{array}$ & $\begin{array}{l}\text { Mild renal } \\
\text { dysfunction }\end{array}$ & $\begin{array}{c}\text { Moderate renal } \\
\text { dysfunction }\end{array}$ & $\begin{array}{l}\text { Severe renal } \\
\text { dysfunction }\end{array}$ & $p$ value \\
\hline Patients, $n$ & 3,441 & 1,649 & 881 & 520 & 343 & \\
\hline Age, years"l & $58 \pm 11$ & $68 \pm 11$ & $71 \pm 10$ & $72 \pm 11$ & $75 \pm 9$ & $<0.001$ \\
\hline Male sex, $n(\%)$ & $3,192(92.8)$ & $967(58.6)$ & $548(62.2)$ & $328(63.1)$ & $174(50.7)$ & $<0.001$ \\
\hline \multicolumn{7}{|l|}{ Comorbidities } \\
\hline Hypertension, $n(\%)$ & $1,288(37.4)$ & $839(50.9)$ & $505(57.3)$ & $320(61.5)$ & $240(70.0)$ & $<0.001$ \\
\hline Diabetes, $n(\%)$ & $751(21.8)$ & $405(24.6)$ & $263(29.9)$ & $203(39.0)$ & $149(43.4)$ & $<0.001$ \\
\hline Current smoker, $n(\%)$ & $2,058(59.8)$ & $570(34.6)$ & $270(30.6)$ & $155(29.8)$ & $73(21.3)$ & $<0.001$ \\
\hline Elevated lipid concentrations ${ }^{\S}, n(\%)$ & $2,418(70.3)$ & $1,108(67.2)$ & $547(62.1)$ & $274(53.0)$ & $174(50.7)$ & $<0.001$ \\
\hline Previous MI, $n(\%)$ & $113(3.3)$ & $74(4.5)$ & $47(5.3)$ & $28(5.4)$ & $21(6.1)$ & $<0.001$ \\
\hline Previous PCI, $n(\%)$ & $163(4.7)$ & $101(6.1)$ & $50(5.7)$ & $35(6.7)$ & $26(7.6)$ & $<0.001$ \\
\hline Previous $C A B G, n(\%)$ & $21(0.6)$ & $16(1.0)$ & $4(0.5)$ & $4(0.8)$ & $6(1.7)$ & $<0.001$ \\
\hline Previous heart failure, $n(\%)$ & $22(0.6)$ & $29(1.8)$ & $35(4.0)$ & $31(6.0)$ & $30(8.7)$ & $<0.001$ \\
\hline Previous stroke, $n(\%)$ & $150(4.4)$ & $123(7.5)$ & $81(9.2)$ & $58(11.2)$ & $54(15.7)$ & $<0.001$ \\
\hline \multicolumn{7}{|l|}{ Initial presentation } \\
\hline ST elevated MI, $n(\%)$ & $2,213(64.7)$ & $1,031(62.9)$ & $552(63.3)$ & $305(59.2)$ & $196(57.6)$ & 0.021 \\
\hline Non-ST elevated MI, $n(\%)$ & $1,205(35.3)$ & $609(37.1)$ & $320(36.7)$ & $210(40.8)$ & $144(42.4)$ & 0.021 \\
\hline Symptom to door time, min"l & $713 \pm 1,346$ & $707 \pm 1,261$ & $761 \pm 1,435$ & $757 \pm 1,230$ & $1,062 \pm 1,614$ & $<0.001$ \\
\hline Pre-hospital resuscitation, $n(\%)$ & $46(1.3)$ & $45(2.7)$ & $28(3.2)$ & $23(4.4)$ & $21(6.1)$ & $<0.001$ \\
\hline Systolic BP, $m m H^{g}$ II & $130 \pm 30$ & $127 \pm 29$ & $124 \pm 42$ & $121 \pm 34$ & $119 \pm 34$ & $<0.001$ \\
\hline Diastolic BP, $m m H g$ II & $80 \pm 22$ & $77 \pm 17$ & $75 \pm 36$ & $73 \pm 19$ & $74 \pm 54$ & $<0.001$ \\
\hline Preinfarct angina, $n(\%)$ & $1,373(40.5)$ & $710(43.8)$ & $359(41.7)$ & $204(40.0)$ & $144(42.9)$ & 0.224 \\
\hline Typical chest pain, $n(\%)$ & $3,043(88.8)$ & $1,397(85.4)$ & $712(81.7)$ & $389(75.2)$ & $238(70.0)$ & $<0.001$ \\
\hline Dyspnea, $n(\%)$ & $732(21.4)$ & $448(27.4)$ & $325(37.4)$ & $218(42.2)$ & $167(49.3)$ & $<0.001$ \\
\hline Killip class $>I, n(\%)$ & $607(18.4)$ & 441 (27.9) & $288(34.4)$ & $198(40.4)$ & $189(58.3)$ & $<0.001$ \\
\hline$Q$ wave infarction, $n(\%)$ & $576(16.6)$ & $236(14.3)$ & $131(14.9)$ & $79(15.2)$ & $62(18.1)$ & 0.133 \\
\hline Atrial fibrillation, $n(\%)$ & $87(2.5)$ & $81(4.9)$ & $53(6.0)$ & $34(6.5)$ & $28(8.2)$ & $<0.001$ \\
\hline Primary VT/VF, $n(\%)$ & $20(0.6)$ & $20(1.2)$ & $9(1.0)$ & $10(1.9)$ & $6(1.7)$ & 0.008 \\
\hline \multicolumn{7}{|l|}{ Laboratory findings } \\
\hline$L V E F<40 \%, n(\%)$ & $372(12.0)$ & $212(14.5)$ & $176(22.8)$ & $127(28.7)$ & $90(31.5)$ & $<0.001$ \\
\hline$e G F R, \mathrm{ml} \cdot \mathrm{min}^{-1} \cdot 1.73 \mathrm{~m}^{-2}$ & $106.4(98.1-115.7)$ & $83.3(79.7-86.6)$ & $68.4(65.0-72.1)$ & $53.3(49.0-56.8)$ & $37.7(34.0-41.3)$ & $<0.001$ \\
\hline Creatinine, $m g / d l^{\ddagger}$ & $0.9(0.9-1.0)$ & $1.1(0.9-1.2)$ & $1.2(1.1-1.3)$ & $1.4(1.3-1.6)$ & $1.8(1.6-1.9)$ & $<0.001$ \\
\hline Troponin $I, n g / m l^{\ddagger}$ & $22.8(5.0-52.2)$ & $18.18(3.2-50.0)$ & $16.5(2.5-50.0)$ & $21.7(3.7-50.0)$ & $14.0(4.2-44.3)$ & $<0.001$ \\
\hline$C K-M B, n g / m l^{+}$ & $94.8(26.0-232.0)$ & $78.7(19.0-219.5)$ & $65.8(16.5-193.3)$ & $47.0(15.0-175.0)$ & $43.3(11.0-158.9)$ & $<0.001$ \\
\hline Total cholesterol, $\mathrm{mg} / \mathrm{dl}^{+}$ & $183.0(157.0-211.0)$ & $181.0(155.0-209.0)$ & $177.0(149.0-209.0)$ & $169.0(141.0-205.0)$ & $167.0(138.3-201.5)$ & $<0.001$ \\
\hline Triglyceride, $m g / d l^{\ddagger}$ & $109.0(72.0-163.0)$ & $101.0(69.0-146.0)$ & $103.0(70.0-149.0)$ & $101.0(71.0-145.0)$ & $96.0(72.0-138.0)$ & $<0.001$ \\
\hline HDL-cholesterol, $\mathrm{mg} / d l^{+}$ & $43.0(37.0-51.0)$ & $44.0(37.0-52.0)$ & $42.0(35.0-50.0)$ & $42.0(35.3-51.0)$ & $41.0(34.0-49.0)$ & $<0.001$ \\
\hline LDL-cholesterol, $\mathrm{mg} / \mathrm{dl}$ & $116.0(94.0-142.0)$ & $115.0(92.0-140.0)$ & $110.0(87.0-136.0)$ & $108.0(84.0-140.0)$ & $104.0(80.0-134.0)$ & $<0.001$ \\
\hline
\end{tabular}

eGFR, estimated glomerular filtration rate; MI, myocardial infarction; PCI, percutaneous coronary intervention; CABG, coronary artery bypass grafting; BP, blood pressure; VT/VF, ventricular tachycardia/ventricular fibrillation; $L V E F$, left ventricular ejection fraction; $C K$, creatine kinase; HDL, high-density lipoprotein; $L D L$, low-density lipoprotein.

IData are presented as the means \pm standard deviation.

Data are presented as the median with $25-75$ percentiles.

$\S$ Defined as total cholesterol level $>240 \mathrm{mg} / \mathrm{dl}$ or $L D L$-cholesterol $>100 \mathrm{mg} / \mathrm{dl}$.

\section{Results}

The median value of estimated GFR and serum creatinine of 6,834 patients were $90.3 \mathrm{ml} \cdot \mathrm{min}^{-1} \cdot 1.73 \mathrm{~m}^{-2}$ (interquartile range: $74.4-106.4$ ) and $1.0 \mathrm{mg} / \mathrm{dl}$ (interquartile range: 0.9 1.2), respectively. Although we enrolled acute MI patients with serum creatinine concentration $\leq 2.0 \mathrm{mg} / \mathrm{dl}$ as an object of study and mild elevation of serum creatinine concentration was observed in just $435(6.4 \%)$ patients, $863(12.6 \%)$ patients had estimated GFR of less than $60 \mathrm{ml} \cdot \mathrm{min}^{-1}$. $1.73 \mathrm{~m}^{-2}$.

\section{Baseline Characteristics}

Baseline demography and clinical characteristics of patients are shown in Table 1 by spectrum of renal function. Old age and female sex were more frequently observed in patients with renal dysfunction. Except current smoker and elevated lipid concentration, a prevalence of cardiovascular comorbidities, such as hypertension, diabetes, and heart failure increased with declining renal function. Patients with renal dysfunction were delayed remarkably in 'onset to door time' and more likely to present with heart failure (Killip class $>\mathrm{I}$ ). These patients had a higher incidence of pre-hospital resuscitation, lower blood pressure, dyspnea, left ventricular (LV) systolic dysfunction, and arrhythmia than patients with preserved renal function. The rates of STEMI and typical chest pain were significantly higher in the patients with preserved renal function.

\section{Reperfusion Therapy and Adjunctive Medical Therapy}

$\mathrm{PCI}$ and $\mathrm{CABG}$ during the index hospitalization were performed in $83.3 \%$ and $3.3 \%$ of total study population, respectively. PCI was used less frequently in patients with moderate and severe renal dysfunction, whereas the use of CABG showed no significant difference among each group (Table 2). We specified acute MI to STEMI and NSTEMI to show an association between primary reperfusion strategies and renal function (Table 2). The use of thrombolytic therapy was significantly lower in patients with moderate and severe renal dysfunction. However, an incidence of primary and facilitated PCI in STEMI was similar across the spectrum of renal function. Patients with preserved renal function who developed NSTEMI were treated more frequently with early invasive PCI. After adjustment for baseline char- 
Table 2 Treatments of Acute MI According to the eGFR

\begin{tabular}{|c|c|c|c|c|c|c|}
\hline & $\begin{array}{l}\text { Normal renal } \\
\text { function }\end{array}$ & $\begin{array}{l}\text { Preserved renal } \\
\quad \text { function }\end{array}$ & $\begin{array}{l}\text { Mild renal } \\
\text { dysfunction }\end{array}$ & $\begin{array}{c}\text { Moderate renal } \\
\text { dysfunction }\end{array}$ & $\begin{array}{l}\text { Severe renal } \\
\text { dysfunction }\end{array}$ & $p$ value \\
\hline \multicolumn{7}{|l|}{ ST elevated MI } \\
\hline Patients, $n$ & 2,213 & 1,031 & 552 & 305 & 196 & \\
\hline Primary PCI, $n(\%)$ & $1,653(73.9)$ & $781(75.1)$ & $423(75.4)$ & $233(75.2)$ & $140(70.4)$ & 0.625 \\
\hline Facilitated PCI, $n(\%)$ & $64(2.9)$ & $30(2.9)$ & $18(3.2)$ & $9(2.9)$ & $5(2.5)$ & 0.989 \\
\hline Thombolytic therapy, $n(\%)$ & $258(11.5)$ & $97(9.3)$ & $34(6.1)$ & $19(6.1)$ & $12(6.0)$ & $<0.001$ \\
\hline Conservative therapy, $n(\%)$ & $286(12.8)$ & $137(13.2)$ & $84(15.0)$ & $47(15.2)$ & $40(21.1)$ & 0.040 \\
\hline \multicolumn{7}{|l|}{ Non-ST elevated MI } \\
\hline Patients, $n$ & 1,205 & 609 & 320 & 210 & 144 & \\
\hline Early invasive therapy, $n(\%)$ & $623(53.8)$ & $274(47.7)$ & $132(43.9)$ & $75(38.5)$ & $45(33.1)$ & $<0.001$ \\
\hline Patients, $n$ & 3,441 & 1,649 & 881 & 520 & 343 & \\
\hline$C A G$, the index hospitalization, $n(\%)$ & $3,151(91.6)$ & $1,449(87.9)$ & $758(86.0)$ & $426(81.9)$ & $257(74.9)$ & $<0.001$ \\
\hline$P C I$, the index hospitalization, $n(\%)$ & $3,054(89.1)$ & $1,387(84.5)$ & $730(83.4)$ & $390(75.4)$ & $231(67.7)$ & $<0.001$ \\
\hline$C A B G$, the index hospitalization, $n(\%)$ & $7(0.2)$ & $3(0.2)$ & $2(0.2)$ & $1(0.2)$ & 0 & 0.625 \\
\hline \multicolumn{7}{|l|}{ Medical therapy } \\
\hline \multicolumn{7}{|l|}{ During the index hospitalization, $n(\%)$} \\
\hline Aspirin & $3,389(98.5)$ & $1,621(98.3)$ & $860(97.6)$ & $498(95.8)$ & $331(96.5)$ & $<0.001$ \\
\hline Thienopyridine & $3,341(97.1)$ & $1,587(96.2)$ & $838(95.1)$ & $483(92.9)$ & $320(93.3)$ & $<0.001$ \\
\hline Cilostazole & $1,191(34.6)$ & $514(31.2)$ & $242(27.5)$ & $137(26.3)$ & $84(24.5)$ & $<0.001$ \\
\hline$\beta$-blocker & $2,532(73.6)$ & $1,147(69.6)$ & $599(68.0)$ & $321(61.7)$ & $211(61.5)$ & $<0.001$ \\
\hline$A C E I / A R B$ & $2,851(82.9)$ & $1,325(80.4)$ & $707(80.2)$ & $390(75.0)$ & $243(70.8)$ & $<0.001$ \\
\hline Nitrate & $2,464(71.6)$ & $1,111(67.4)$ & $588(66.7)$ & $330(63.5)$ & $211(61.5)$ & $<0.001$ \\
\hline Calcium channel blocker & $492(14.3)$ & $237(14.4)$ & $127(14.4)$ & $82(15.8)$ & $66(19.2)$ & 0.149 \\
\hline Statin & $2,718(79.0)$ & $1,237(75.0)$ & $629(71.4)$ & $346(66.5)$ & $232(67.6)$ & $<0.001$ \\
\hline Unfractionated heparin & $1,981(57.6)$ & $862(52.3)$ & $467(53.0)$ & $261(50.2)$ & $176(51.3)$ & $<0.001$ \\
\hline$L M W H$ & $1,257(36.5)$ & $623(37.8)$ & $311(35.3)$ & $189(36.3)$ & $123(35.9)$ & 0.785 \\
\hline Glycoprotein IIb/III inhibitor & $433(12.6)$ & $202(12.2)$ & $114(12.9)$ & $56(10.8)$ & $29(8.5)$ & 0.171 \\
\hline \multicolumn{7}{|l|}{ After discharge, $n(\%)$} \\
\hline Aspirin & $3,179(92.4)$ & $1,457(88.4)$ & $747(84.8)$ & $410(78.8)$ & $248(72.3)$ & $<0.001$ \\
\hline Thienopyridine & $3,059(88.9)$ & $1,381(83.7)$ & $711(80.7)$ & $376(72.3)$ & $236(68.8)$ & $<0.001$ \\
\hline$\beta$-blocker & $2,329(67.7)$ & $1,040(63.1)$ & $535(60.7)$ & $283(54.4)$ & $164(47.8)$ & $<0.001$ \\
\hline$A C E I / A R B$ & $2,702(78.5)$ & $1,206(73.1)$ & $616(69.9)$ & $331(63.7)$ & $200(58.3)$ & $<0.001$ \\
\hline Statin & $2,579(74.9)$ & $1,163(70.5)$ & $569(64.6)$ & $287(55.2)$ & $182(53.1)$ & $<0.001$ \\
\hline
\end{tabular}

CAG, coronary angiography; ACEI/ARB, angiotensin-converting enzyme inhibitor/angiotensin receptor blocker; LMWH, low molecular weight heparin. Other abbreviations see in Table 1 .

Table 3 Determinants of Primary Reperfusion Therapy

\begin{tabular}{|c|c|c|c|c|}
\hline \multirow{2}{*}{ Adjusted results } & \multicolumn{2}{|c|}{ ST elevated MI } & \multicolumn{2}{|c|}{ Non-ST elevated MI } \\
\hline & OR $(95 \% C I)$ & $p$ value & OR $(95 \% C I)$ & $p$ value \\
\hline Chest pain & $1.9(1.4-2.5)$ & $<0.001$ & $2.9(2.3-3.8)$ & $<0.001$ \\
\hline Dyspnea & $0.7(0.6-1.0)$ & 0.028 & & \\
\hline$Q$ wave on initial $E C G$ & $0.4(0.3-0.5)$ & $<0.001$ & & \\
\hline Previous stroke & $0.5(0.3-0.8)$ & 0.002 & & \\
\hline Malignancy & $0.3(0.2-0.7)$ & 0.002 & & \\
\hline Symptom to door time, min & $1.1(1.0-1.3)$ & $<0.001$ & & \\
\hline Female sex & & & $0.7(0.5-1.0)$ & 0.031 \\
\hline$L V E F<40 \%$ & & & $1.9(1.3-2.6)$ & $<0.001$ \\
\hline Elevated lipid concentrations & & & $1.5(1.1-2.0)$ & 0.003 \\
\hline
\end{tabular}

Multivariate logistic regression model. Candidates for adjustment: age, sex, weight, abdominal circumference, previous MI, diabetes, current smoker, previous stroke, chronic lung disease, previous heart failure, cancer, symptom at presentation, Killip class $>I, Q$ wave and bundle branch block on initial electrocardiogram, $L V E F<40 \%$, spectrum of renal function, 'symptom to door time', heart rate, serum creatinine concentration, $C K-M B$.

OR, odds ratio; CI, confidence interval; ECG, electrocardiogram. Other abbreviations see in Table 1.

acteristics, renal function was not presented as a determinant of primary reperfusion strategy in STEMI and early invasive therapy in NSTEMI (Table 3).

Adjunctive medical therapy was recorded and analyzed (Table 2). Standard medications such as antiplatelet agents, $\beta$-blocker, statin, unfractionated heparin and angiotensinconverting enzyme (ACE) inhibitor/angiotensin-receptor blocker (ARB) during the index hospitalization were used less frequently in patients with renal dysfunction. These trends were persisted with medical therapy at hospital discharge.

\section{Angiographic Findings}

Angiographic findings are shown in Table 4. CAG was performed in $6,041(88.4 \%)$ of total study population and patients with renal dysfunction were less likely to undergo CAG. Of infarct-related arteries, right coronary artery (RCA) and left main trunk increased with declining renal function. Multivessel disease on CAG had remarkably high incidence in patients with renal dysfunction. However, complex lesion (type C) and Thrombolysis In Myocardial Infarction (TIMI) flow of grade 0 at initial CAG had fairly similar incidence among each group. 
Table 4 CAG and PCI According to the eGFR

\begin{tabular}{|c|c|c|c|c|c|c|}
\hline & $\begin{array}{l}\text { Normal renal } \\
\text { function }\end{array}$ & $\begin{array}{l}\text { Preserved renal } \\
\text { function }\end{array}$ & $\begin{array}{c}\text { Mild renal } \\
\text { dysfunction }\end{array}$ & $\begin{array}{c}\text { Moderate renal } \\
\text { dysfunction }\end{array}$ & $\begin{array}{c}\text { Severe renal } \\
\text { dysfunction }\end{array}$ & $p$ value \\
\hline \multicolumn{7}{|l|}{ CAG during the index hospitalization } \\
\hline Patients, $n$ & 3,151 & 1,449 & 758 & 426 & 257 & \\
\hline Diseased vessel $>1, n(\%)$ & $1,665(52.9)$ & $879(60.5)$ & $507(67.0)$ & 307 (71.9) & $189(73.8)$ & $<0.001$ \\
\hline Target vessel, $n(\%)$ & & & & & & $<0.001$ \\
\hline Left anterior descending & $1,606(51.0)$ & $698(48.2)$ & $326(43.0)$ & $192(45.1)$ & $100(38.9)$ & \\
\hline Left circumflex & $521(16.5)$ & $223(15.4)$ & $128(16.9)$ & $62(14.6)$ & $25(9.7)$ & \\
\hline Right coronary & $982(31.2)$ & $496(34.2)$ & $284(37.5)$ & $161(37.8)$ & $119(46.3)$ & \\
\hline Left main & $42(1.3)$ & $32(2.2)$ & $20(2.6)$ & $11(2.6)$ & $13(5.1)$ & \\
\hline Lesion type $C, n(\%)$ & $1,456(49.2)$ & $718(52.4)$ & $383(53.6)$ & $219(54.6)$ & $132(53.9)$ & 0.098 \\
\hline TIMI flow grade 0 at initial CAG, $n(\%)$ & $1,319(43.1)$ & $648(45.9)$ & $312(42.3)$ & $189(46.6)$ & $121(48.2)$ & 0.314 \\
\hline \multicolumn{7}{|l|}{ PCI during the index hospitalization } \\
\hline Patients, $n$ & 3,054 & 1,387 & 730 & 390 & 231 & \\
\hline Stent implantation, $n(\%)$ & $2,790(91.2)$ & $1,242(89.3)$ & $651(89.2)$ & $353(90.3)$ & $213(92.2)$ & 0.160 \\
\hline Kind of implanted stents, $n(\%)$ & & & & & & 0.265 \\
\hline Sirolimus eluting stent & $1,342(48.6)$ & $586(48.1)$ & $306(47.8)$ & $165(47.4)$ & $94(44.8)$ & \\
\hline Paclitaxel eluting stent & $928(33.6)$ & $391(32.1)$ & $211(33.0)$ & $120(34.5)$ & $81(38.6)$ & \\
\hline Other drug-eluting stent & $284(10.3)$ & $167(13.7)$ & $74(11.6)$ & $38(10.9)$ & $17(8.1)$ & \\
\hline Bare metal stent & $208(7.5)$ & $74(6.1)$ & $49(7.7)$ & $25(7.2)$ & $18(8.6)$ & \\
\hline Stent length $>30 \mathrm{~mm}, n(\%)$ & $702(25.5)$ & $333(27.3)$ & $192(30.0)$ & $90(25.8)$ & $59(28.0)$ & 0.172 \\
\hline Stent diameter $\leq 3.0 \mathrm{~mm}, n(\%)$ & $514(18.6)$ & $259(21.2)$ & $163(25.5)$ & $80(23.0)$ & $65(30.8)$ & $<0.001$ \\
\hline Multiple stents, $n(\%)$ & $932(34.2)$ & $422(35.2)$ & $260(41.0)$ & $130(38.6)$ & $78(37.7)$ & 0.016 \\
\hline Final TIMI flow grade $3, n(\%)$ & $2,778(94.2)$ & $1,234(92.0)$ & $653(92.4)$ & $339(88.7)$ & $213(90.6)$ & 0.004 \\
\hline PCI-related complication, $n(\%)$ & $275(8.1)$ & $230(14.2)$ & $168(19.4)$ & $111(21.7)$ & $111(33.1)$ & $<0.001$ \\
\hline
\end{tabular}

TIMI, Thrombolysis In Myocardial Infarction. Other abbreviations see in Table 1.

Table 5 Outcome Data According to the eGFR

\begin{tabular}{|c|c|c|c|c|c|c|}
\hline & $\begin{array}{l}\text { Normal renal } \\
\text { function }\end{array}$ & $\begin{array}{l}\text { Preserved renal } \\
\quad \text { function }\end{array}$ & $\begin{array}{l}\text { Mild renal } \\
\text { dysfunction }\end{array}$ & $\begin{array}{c}\text { Moderate renal } \\
\text { dysfunction }\end{array}$ & $\begin{array}{l}\text { Severe renal } \\
\text { dysfunction }\end{array}$ & $p$ value \\
\hline Patients, $n$ & 3,441 & 1,649 & 881 & 520 & 343 & \\
\hline In-hospital Death, $n(\%)$ & $45(1.3)$ & $63(3.8)$ & $65(7.4)$ & $57(10.9)$ & $58(16.9)$ & $<0.001$ \\
\hline Cardiac death, $n(\%)$ & $32(0.9)$ & $51(3.1)$ & $56(6.4)$ & $47(9.0)$ & $47(13.7)$ & $<0.001$ \\
\hline Total MACE at 180 days, $n(\%)$ & $166(4.8)$ & $144(8.7)$ & $117(13.3)$ & $83(16.0)$ & $84(24.5)$ & $<0.001$ \\
\hline Overall mortality, $n(\%)$ & $53(1.5)$ & $85(5.2)$ & $82(9.3)$ & $70(13.5)$ & $69(20.1)$ & $<0.001$ \\
\hline Cardiac mortality, $n(\%)$ & $38(1.1)$ & $65(3.9)$ & $67(7.6)$ & $58(11.2)$ & $53(15.5)$ & $<0.001$ \\
\hline Recurrent $M I, n(\%)$ & $24(0.7)$ & $15(0.9)$ & $10(1.1)$ & $7(1.4)$ & $14(4.1)$ & $<0.001$ \\
\hline Repeated PCI, $n(\%)$ & $90(2.6)$ & $47(2.9)$ & $27(3.1)$ & $11(2.1)$ & $9(2.6)$ & 0.137 \\
\hline$C A B G, n(\%)$ & $19(0.5)$ & $11(0.6)$ & $5(0.6)$ & $3(0.6)$ & $1(0.3)$ & 0.627 \\
\hline
\end{tabular}

MACE, major cardiac adverse event. Other abbreviations see in Table 1.

Of patients who underwent PCI, plain balloon angioplasty and stent implantation were conducted in $442(7.8 \%)$ and $5,249(92.2 \%)$ patients, respectively. The rates of stent implantation were similar across the spectrum of renal function. Bare metal stent, sirolimus-eluting stent $\left(\right.$ Cypher ${ }^{\circledR}$, Cordis, Johnson \& Johnson, New Brunswick, NJ, USA), paclitaxel-eluting stent (Taxus ${ }^{\circledR}$, Boston Scientific, Boston, MA, USA) and other drug-eluting stents were analyzed and compared with implanted stent during PCI, but there was no significant differences according to type of stent in among each group. The use of stent with small diameter $(\leq 3 \mathrm{~mm})$ was increased with declining renal function. Final TIMI flow of grade 3, as index of successful result of PCI, was obtained less frequently in the patients with moderate renal dysfunction. PCI-related complications including cardiogenic shock needing intra-aortic balloon counter-pulsation, heart failure class III or IV, secondary ventricular tachyarrhythmia, major bleeding and stroke had 4-fold increment of incidence rates between patients with normal renal function and those with severe renal dysfunction.
Clinical Outcomes According to Renal Function and Predictors of Clinical Outcomes

Of the total 6,834 patients, in-hospital mortality was recorded in $288(4.3 \%)$ patients. Long-term mortality and adverse events were developed in 359 (5.3\%) and 594 $(8.7 \%)$ patients, respectively.

During the index hospitalization, overall mortality was observed more frequently in patients with renal dysfunction (Table 5). These findings were persisted in long-term mortality and adverse events. Increments of long-term mortality and adverse event rates began even at patients with mild renal dysfunction as compared with those with normal renal function (Fig 1). A stepwise increment of risk ratio for mortality and adverse events according to renal function was shown at Table 6. However, statistical significance of increased risk ratio for in-hospital mortality was observed in just 2 groups with moderate and severe renal dysfunction. Also, only 1 group of severe renal dysfunction had significance in increased risk ratio for long-term mortality and adverse events.

Predictors of in-hospital mortality were diabetes, lung disease, Killip class $>$ I, LVEF $<40 \%$, and moderate and severe renal dysfunction (Table 7). Prescription of $\beta$-blocker and 
ACE inhibitor/ARB improved survivals during hospitalization. Factors associated with an increased risk for long-term mortality included advanced age, previous MI, Killip class $>$ I and LVEF $<40 \%$, PCI-related complications and severe renal dysfunction. RCA infarction and the use of statin (in-hospital), thienopyridine and $\beta$-blocker reduced longterm mortality. The risk for long-term adverse events was increased by severe renal dysfunction, multi-vessel disease, previous PCI and PCI-related complication.

\section{Discussion}

\section{Serum Creatinine and Estimated GFR in Acute MI}

Although enrolled patients have serum creatinine concentrations of $\leq 2.0 \mathrm{mg} / \mathrm{dl}$ as mild degree renal impairment, this work shows that renal function by estimated GFR has broad spectrum, and some patients have moderate or severe renal impairments. Our finding is consistent with some previous studies. With comparison between serum creatinine and estimated GFR, the incidence of renal impairment has been underestimated by serum creatinine in same populations with acute MI2,14 For example, Gibson et al showed that $74 \%$ of patients had a normal creatinine concentration of $1.2 \mathrm{mg} / \mathrm{dl}$ or less; however, nearly $60 \%$ had any degree of renal dysfunction (estimated GFR $\leq 90 \mathrm{ml} \cdot \mathrm{min}^{-1} \cdot 1.73 \mathrm{~m}^{-2}$ ) with $20 \%$ having moderate or severe renal dysfunction! ${ }^{14}$ Also, previous studies showed that the incidence of renal impairment, reflected by estimated GFR, was remarkably high in patients with acute MI 1,15 This observation seems to be important because we should avoid underscoring renal function by solely use of serum creatinine in real practice. Consequently, physicians have to rely on estimated GFR, as an index of renal function, rather than serum creatinine concentrations in patients with acute MI.

\section{Renal Function and Clinical Outcomes After Acute MI}

Furthermore, the present study shows that estimated GFR has a significant impact on survival rates after acute MI, even in patients with normal or mildly elevated value of serum creatinine. The gradient of long-term survival rates is observed in patients with mild renal dysfunction. We observe a similar gradient of rates in overall adverse events. This finding is consistent with previous works showing greater mortality rates in severe and end-stage renal disease!4,16-18 Explanations for more adverse outcomes have included several factors such as advanced age, excessive comorbidities, special pathophysiologic factors and 'therapeutic nihilism' associated with renal dysfunction, $6,17,19$ These associations are confirmed and extended in our study by showing other clinical factors as follows: (1) remarkably higher incidence of hypertension, diabetes, stroke and previous heart failure; (2) underuse of reperfusion therapies
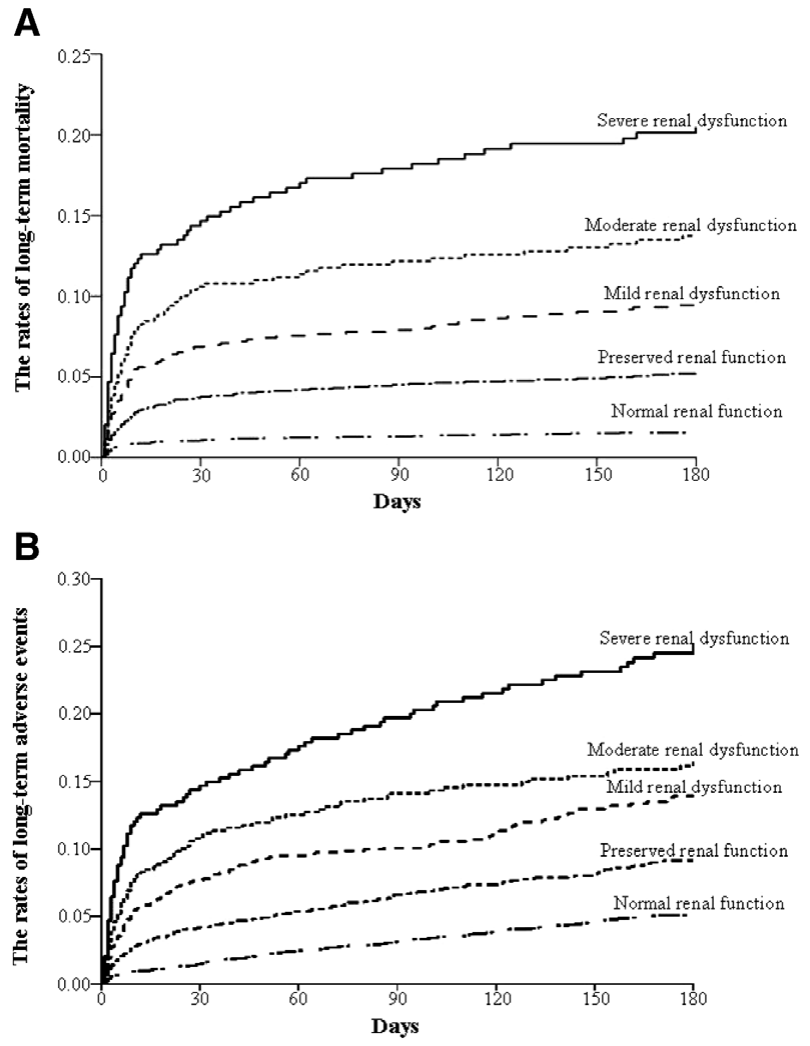

Fig 1. Life table estimates of the rates of long-term mortality (A) and of long-term adverse event (B) according to spectrum of renal function at 180 days.

and standard medications; (3) serious angiographic findings; and (4) development of more PCI-related complications with declining renal function.

With other conventional risk predictors, we identify moderate and severe renal dysfunction as the most significant risk predictor for in-hospital mortality after adjustment for confounding factors. However, only severe renal dysfunction is represented as a prognostic factor in long-term mortality and adverse outcome. This might be explained by the specific pathologic conditions playing an important role over a longer period of time, in patients with severe renal dysfunction. These include a greater comorbidity and accelerated atherosclerosis by enhanced oxidation of low-density lipoprotein-cholesterol, endothelial dysfunction and pathologic arterial calcification!,6,20 Previous investigators reported that these potential mechanisms attribute to complications from acute MI'1,22 In this work, the proportion of PCI-related complications has abrupt increase between

Table 6 The Increased Risk for Death and MACE After MI According to the eGFR

\begin{tabular}{|c|c|c|c|c|c|}
\hline Adjusted results & $\begin{array}{l}\text { Severe renal } \\
\text { dysfunction }\end{array}$ & $\begin{array}{l}\text { Moderate renal } \\
\text { dysfunction }\end{array}$ & $\begin{array}{l}\text { Mild renal } \\
\text { dysfunction }\end{array}$ & $\begin{array}{l}\text { Preserved renal } \\
\quad \text { function }\end{array}$ & Reference $^{\ddagger}$ \\
\hline In-hospital mortality $\#$ OR $(95 \% C I)$ & $7.0(3.2-18.2)$ & $5.2(2.1-13.5)$ & $2.2(0.7-6.7)^{\S}$ & $1.5(0.5-4.3)^{\S}$ & 1.0 \\
\hline Long-term mortality $\mathrm{HR}(95 \% \mathrm{CI})$ & $4.2(1.6-10.8)$ & $1.5(0.6-3.5)^{\S}$ & $1.3(0.5-3.4)^{\S}$ & $0.8(0.3-1.8)^{\S}$ & 1.0 \\
\hline Long-term adverse events $\mathrm{HR}(95 \% \mathrm{CI})$ & $4.0(2.3-7.1)$ & $1.5(0.9-2.6)^{\S}$ & $1.4(0.9-2.3)^{\S}$ & $1.0(0.7-1.5)^{\S}$ & 1.0 \\
\hline
\end{tabular}

HR, hazard ratio. Other abbreviations see in Tables 1,3,5.

"Multivariate logistic regression model.

Cox proportional-hazards modeling.

sp-value is more than 0.05 for comparisons among all groups and for comparisons between individual groups.

$\$$ Reference indicates normal renal function group. 
Table 7 Predictors of Clinical Outcomes

\begin{tabular}{|c|c|c|c|c|c|c|}
\hline \multirow{2}{*}{ Adjusted results } & \multicolumn{2}{|c|}{ In-hospital mortality } & \multicolumn{2}{|c|}{ Long-term mortality ${ }^{\dagger}$} & \multicolumn{2}{|c|}{ Long-term adverse event ${ }^{\dagger}$} \\
\hline & OR $(95 \% C I)$ & $p$ value & $H R(95 \% C I)$ & $p$ value & $H R(95 \% C I)$ & $p$ value \\
\hline Moderate renal dysfunction & $5.2(2.1-13.5)$ & 0.005 & & & & \\
\hline Severe renal dysfunction & $7.0(3.2-18.2)$ & 0.001 & $4.2(1.6-10.8)$ & 0.003 & $4.0(2.3-7.1)$ & $<0.001$ \\
\hline$L V E F,<40 \%$ & $3.4(1.7-6.8)$ & 0.001 & $1.8(1.0-3.1)$ & 0.040 & & \\
\hline Killip class $>I$ & $2.8(1.3-5.7)$ & 0.007 & $2.4(1.3-4.6)$ & 0.005 & & \\
\hline Age & & & $1.1(1.0-1.3)$ & $<0.001$ & & \\
\hline Diabetes & $2.2(1.1-4.6)$ & 0.033 & & & & \\
\hline Chronic lung disease & $4.2(1.2-15.0)$ & 0.026 & & & & \\
\hline Previous MI & & & $3.4(1.6-7.1)$ & 0.001 & & \\
\hline Previous PCI & & & & & $2.5(1.4-4.8)$ & 0.003 \\
\hline Diseased vessel $>1$ & & & & & $1.9(1.3-2.7)$ & $<0.001$ \\
\hline$R C A$ infarction & & & $0.5(0.2-0.9)$ & 0.014 & & \\
\hline Primary $V T / V F$ & $3.5(1.5-7.9)$ & 0.003 & & & & \\
\hline PCI-related complications & & & $6.2(3.4-11.5)$ & $<0.001$ & $2.2(1.6-3.2)$ & $<0.001$ \\
\hline Aspirin (in-hospital) & & & & & $0.3(0.1-0.7)$ & 0.011 \\
\hline ß-blocker (in-hospital) & $0.3(0.1-0.6)$ & 0.001 & & & & \\
\hline ACEI/ARB (in-hospital) & $0.3(0.2-0.7)$ & 0.005 & & & & \\
\hline Statin (in-hospital) & & & $0.4(0.2-0.6)$ & $<0.001$ & $0.6(0.5-0.9)$ & 0.016 \\
\hline Aspirin at discharge & & & & & $0.5(0.2-1.0)$ & 0.040 \\
\hline Thionepyridine at discharge & & & $0.1(0.05-0.2)$ & $<0.001$ & $0.3(0.1-0.5)$ & $<0.001$ \\
\hline$\beta$-blocker at discharge & & & $0.2(0.1-0.4)$ & $<0.001$ & & \\
\hline
\end{tabular}

$R C A$, right coronary artery. Other abbreviations see in Tables 1-3,6.

\#Multivariate logistic regression model.

Cox proportional-hazards modeling.

severe and moderate renal dysfunction, and these complications are one of the important predictors in long-term mortality.

\section{Renal Function and Use of Medical Therapy}

Previous studies which performed in randomized controlled trials have shown underuse of standard medications in patients with renal dysfunction? ${ }^{23-25}$ This work confirms those findings in unselected patients, despite considerable advances of modern pharmaceutical treatment in acute MI. We show that, as well as thrombolytic agent, medications with risk benefit after MI, are used less frequently during the index hospitalization and follow-up periods with declining renal function. Also, several medications have a significant impact on risk reduction of mortality and adverse outcome. The use of aspirin, thienopyridine, statin, $\beta$-blocker and ACE inhibitor have a favorable benefit to risk ratio for mortality and adverse events across the spectrum of renal function.

McCullough et al reported that prescription of standard medications is limited by lack of evidence in patients with renal dysfunction, because these patients have been excluded from randomized trials $2^{26}$ In real practice, physicians might be reluctant to prescribe drugs such as ACE inhibitors and $\beta$-blockers for patients with some degree of renal dysfunction because of the concern that they may worsen renal function. Also, aspirin and thienopyridine in those patients are commonly withheld as a result of increased risk for bleeding. However, it has been shown that the reduction of mortality after acute MI by prescription of these drugs might be even greater in high-risk patients.5 Some studies have shown that ACE inhibitors and $\beta$-blockers have a survival benefit in acute MI patients with LV dysfunction, even in the presence moderate renal dysfunction ${ }^{25}$ Recently, in 2006, Hou et al reported the safety and utility of ACE inhibitor in advanced renal dysfunction, stage III and IV? 7 We suggest that more aggressive prescription of proven medications should make survival rates even in patients with severe renal dysfunction to be improved.

\section{Renal Function and the Use of Reperfusion Strategy}

Previous studies have shown that coronary reperfusion therapies during the index hospitalization are used less frequently in patients with renal dysfunction who develop acute MI ${ }^{17,22,23}$ As a medical treatment, this is partly associated with advanced age, increased comorbidity and more compromised hemodynamic status in decreased renal function ${ }^{26}$ Wright et al described that patients with any degree of renal dysfunction are more likely to have contraindications to thrombolytic therapy than those without renal dysfunction.8 We partly ascertained previous works; however, primary and facilitated PCIs in patients with STEMI are performed with similar rate among each group. When acute MI is specified to STEMI and NSTEMI, renal function does not seem to be considered as determinant of early and primary reperfusion therapy in both subgroups of acute MI. As compared with early reports, which described primary reperfusion therapy, we think more aggressive therapy has been accomplished, at least in primary reperfusion regardless of renal function.

It has not been conclusive that PCI reduces risk ratio of mortality after acute MI in populations with lower spectrum of renal function. Al Suwaidi et al showed that invasive reperfusion therapy, PCI and CABG, is not a significant predictor of 30-day mortality model22 However, other studies reported that reperfusion therapies have the benefits to risk for long-term mortality and improve survival rates! ${ }^{16,28}$ In this work, we found that reperfusion therapies represent no impacts on risk for mortality, despite remarkable advances in PCI and more aggressive approach. We cannot fully explain why these strategies are not associated with risk reduction. It is partly explained by some factors that PCIrelated complications might counterbalance the benefits from early reperfusion ${ }^{28}$ Also, contrast induced worsening renal function and volume status during and after PCI might be attributed to those results. ${ }^{29}$ Dragu et al reported that survival rates in patients who underwent primary PCI are the lowest as compared with those with thrombolysis and even in those without reperfusion therapy 30 We think 
that a randomized trial is needed to elucidate the impact of primary PCI on clinical outcome in acute MI patients with renal dysfunction.

We extend previous work by evaluating the factors related with CAG and PCI according to the spectrum of renal function. Of these factors, multivessels disease and RCA infarction increase risk of long-term adverse events. Coronary stents, especially drug-eluting stent, are used mainly in PCI rather than plain balloon angioplasty in most patients. Inaguma et al showed that stent implantation is associated with risk reduction of overall mortality ${ }^{21}$ However, this work shows no impact on prognosis of stent implantation, even with a drug-eluting stent. This disparate finding might be a result from differences of study population. They enrolled patients with angina pectoris or MI, who underwent PCI. The success of PCI is less likely to be obtained and PCI related complications are more likely to be developed with declining renal function. It seems that these findings may be attribute to diminishing the effect of PCI in mortality and adverse events in this work.

\section{Study Limitations}

This study has some limitations. First, this work has a potential bias because we excluded patients with serum creatinine of more than $2.0 \mathrm{mg} / \mathrm{dl}$ and previous renal disease such as renal failure on dialysis. This exclusion might reduce the strength of risk associated with renal dysfunction. Second, GFR is not measured directly and measured by MCQ equation which has not been tested in oriental populations. Also, GFR might be affected by compromised hemodynamic status because creatinine concentration incorporated in MCQ is initial value. Finally, long-term adverse events including revascularization might be underestimated because we could not perform follow-up CAG in patients without symptoms.

\section{Conclusion}

Although serum creatinine concentrations of patients with acute MI did not exceed $2.0 \mathrm{mg} / \mathrm{dl}$, the present study showed that renal function, which is presented by estimated GFR, has broad spectrums. The current study also showed that the mortality rates are strongly affected by the spectrum of renal function. Also, we confirmed 'therapeutic nihilism' in patients with any degree of renal dysfunction, although life-saving medications had a benefit to risk ratio. Several clinical factors including higher prevalence of comorbidities, compromised hemodynamic status, serious angiographic findings and complications might attribute to these association. Even though patients with acute MI have normal or mildly elevated serum creatinine concentrations, renal function must be reflected by GFR and be considered as an independent risk factor in practice. Also, the constructive utilization of standard treatments for acute MI might be associated with better prognosis in patients with any degree of renal dysfunction.

\section{Acknowledgement}

This study was performed with the support of the Korean Circulation Society (KCS) in the memorandum of the $50^{\text {th }}$ Anniversary of KCS.

\section{References}

1. Anavekar NS, Mcmurray JJ, Velazquez EJ, Solomon SD, Kober L, Rouleau JL, et al. Relation between renal dysfunction and cardiovascular outcomes after myocardial infarction. N Engl J Med 2004; 351:
$1285-1295$.

2. Shlipak MG, Heidenreich PA, Noguchi H, Chertow GM, Browner WS, McClellan MB. Association of renal insufficiency with treatment and outcomes after myocardial infarction in elderly patients. Ann Intern Med 2002; 137: 555-562.

3. Smith GL, Shilipak MG, Havranek EP, Foody JM, Masoudi FA, Rathore SS, et al. Serum urea nitrogen, creatinine, and estimators of renal function: Mortality in older patients with cardiovascular disease. Arch Intern Med 2006; 166: 1134-1142.

4. Herzog CA, Ma JZ, Collins AJ. Poor long-term survival after acute myocardial infarction among patients on long-term dialysis. $N$ Engl $J$ Med 1998; 339: 799-805.

5. Chertow GM, Normand SL, Silva LR, McNeil BJ. Survival after acute myocardial infarction in patients with end-stage renal disease: Results from the cooperative cardiovascular project. Am J Kidney Dis 2000; 35: 1044-1051.

6. McCullough PA. Cardiorenal risk: An important clinical intersection. Rev Cardiovasc Med 2002; 3: 71-76.

7. Landray MJ, Thambyrajah J, McGlynn FJ, Jones HJ, Baigent C, Kendall MJ, et al. Epidemiological evaluation of known and suspected cardiovascular risk factors in chronic renal impairment. Am J Kidney Dis 2001; 38: 537-546.

8. Henry RMA, Kostense PJ, Bos G, Dekker JM, Nijpels G, Heine RJ, et al. Mild renal insufficiency is associated with increased cardiovascular mortality: The Hoorn Study. Kidney Int 2002; 62: 1402-1407.

9. Lee KH, Jeong MH, Ahn YK, Kim JH, Chae SC, Kim YJ, et al; and other Korea Acute Myocardial infarction Registry Investigators. Sex differences of the clinical characteristics and early management in the Korea Acute Myocardial Infarction Registry. Korean Circ J 2007; 37: 64-71.

10. Clase CM, Kiberd BA, Garg AX. Relationship between glomerular filtration rate and the prevalence of metabolic abnormalities: Results from the Third National Health and Nutrition Examination Survey (NHANES III). Nephron Clin Pract 2007; 105: 178-184.

11. French JK, White HD. Clinical implications of the new definition of myocardial infarction. Heart 2004; 90: 99-106.

12. Rigalleau V, Lasseur C, Raffaitin C, Perlemoine C, Barthe N, Chauveau P, et al. The Mayo Clinic Quadratic Equation improves the prediction of glomerular filtration rate in diabetic subjects. Nephrol Dial Transplant 2007; 22: 813-818.

13. Hillege HL, Nitsch D, Pfeffer A, Swedberg K, McMurray JJV, Yusuf $\mathrm{S}$, et al. Renal function as a predictor of outcome in a broad spectrum of patients with heart failure. Circulation 2006; 113: 671-678.

14. Gibson CM, Pinto DS, Murphy SA, Morrow DA, Hobbach HP, Wiviott SD, et al. Association of creatinine and creatinine clearance on presentation in acute myocardial infarction with subsequent mortality. J Am Coll Cardiol 2003; 42: 1535-1543.

15. Zhang Q, Ma CS, Nie SP, Du X, Lv Q, Kang JP, et al. Prevalence and impact of renal insufficiency on clinical outcomes of patients undergoing coronary revascularization. Circ J 2007; 71: 1299-1304.

16. Keeley EC, Kodakia R, Soman S, Borzak S, McCullough PA. Analysis long-term survival after revascularization in patients with chronic kidney disease presenting with acute coronary syndromes. Am J Cardiol 2003; 92: 509-514.

17. Santopinto JJ, Fox KAA, Goldberg RJ, Budaj A, Piñero G, Avezum A, et al. Creatinine clearance and adverse hospital outcomes in patients with acute coronary syndromes: Findings from the global registry of acute coronary events (GRACE). Heart 2003; 89: $1003-1008$.

18. Pitsavos C, Kurlaba G, Panagiotakos DB, Kogias Y, Mantas Y, Chrysohoou C, et al. Association of creatinine clearance and in-hospital mortality in patients with acute coronary syndromes: The GREECS study. Circ J 2007; 71: 9-14.

19. Beattie JN, Soman SS, Sandberg KR, Yee J, Borzak S, Garg M, et al. Determinants of mortality after myocardial infarction in patients with advanced renal dysfunction. Am J Kidney Dis 2001; 37: 1191-1200.

20. Luft FC. Renal disease as a risk factor for cardiovascular disease. Basic Res Cardiol 2000; 95(Suppl): I72-I76.

21. Inaguma D, Tatematsu M, Shinjo H, Suzuki S, Mishima T, Inaba S, et al. Relationship between renal function at the time of percutaneous coronary intervention and prognosis in ischemic heart disease patients. Clin Exp Nephrol 2007; 11: 56-60.

22. Al Suwaidi JA, Reddan DN, Williams K, Pieper KS, Harrington RA, Califf RM, et al. Prognostic implications of abnormalities in renal function in patients with acute coronary syndromes. Circulation 2002; 106: 974-980.

23. Koganei H, Kasanuki H, Ogawa H, Tsurumi Y. Association of glomerular filtration rate with unsuccessful primary percutaneous coronary intervention and subsequent mortality in patients with acute myocardial infarction: From the HIJAMI registry. Circ J 2008; 72: $179-185$. 
24. McCullough PA, Sandberg KR, Borzak S, Hudson MP, Garg M, Manley HJ. Benefits of aspirin and $\beta$-blockade after myocardial infarction in patients with chronic kidney disease. Am Heart J 2002; 144: $226-232$.

25. Shlipak MG, Browner WS, Noguchi H, Massie B, Frances CD, McClellan M. Comparison of the effects of angiotensin-converting enzyme inhibitors and beta blockers on survival in elderly patients with reduced left ventricular function after myocardial infarction. $\mathrm{Am}$ $J$ Med 2001; 110: 425-433.

26. McCullough PA. Acute coronary syndromes in patients with renal failure. Curr Cardiol Rep 2003; 5: 266-270.

27. Hou FF, Zhang X, Zhang GH, Xie D, Chen PY, Zhang WR, et al. Efficacy and safety of benazepril for advanced chronic renal insufficiency. $N$ Engl J Med 2006; 354: 131-140.

28. Wright RS, Reeder GS, Herzog CA, Albright RC, Williams BA, Dvorak DL, et al. Acute myocardial infarction and renal dysfunction: A high-risk combination. Ann Intern Med 2002; 137: 563 -570.

29. Sadeghi HM, Stone GW, Grines CL, Mehran R, Dixon SR, Lansky $\mathrm{AJ}$, et al. Impact of renal insufficiency in patients undergoing primary angioplasty for acute myocardial infarction. Circulation 2003; 108: $2769-2775$.

30. Dragu R, Hehar S, Sandach A, Boyko V, Kapeliovich M, Rispler S, et al. Should primary percutaneous coronary intervention be the preferred method of reperfusion therapy for patients with renal failure and ST-elevation acute myocardial infarction? Am J Cardiol 2006; 97: $1142-1145$

\section{Appendix 1}

Korea Acute Myocardial Infarction Registry Investigators Bon-Kwon Koo, MD; Byung-Ok Kim, MD; Chong-Jin Kim, MD; ChongYun Rhim, MD; Dong-Hoon Choi, MD; Dong-Kyu Jin, MD; Doo-Il Kim, MD; Hyeon-Cheol Gwon, MD; In-Ho Chae, MD; In-Whan Seong, MD; Jae-Young Rhew, MD; Jang-Ho Bae, MD; Jei-Keon Chae, MD; JeongGwan Cho, MD; Jin-Man Cho, MD; Jin-Yong Hwang, MD; Jong-Hyun Kim, MD; Jong-Seon Park, MD; Jung-Han Yoon, MD; Ju-Young Yang, MD; Kee-Sik Kim, MD; Keum-Soo Park, MD; Ki-Bae Seung, MD; KyooRok Han, MD; Kyoung-Tae Jeong, MD; Moo-Hyun Kim, MD; MyeongChan Cho, MD; Myung-Ho Jeong, MD; Myoung-Yong Lee, MD; NaeHee Lee, MD; Seok-Kyu Oh, MD; Seong-Wook Park, MD; Seong-Woon Rha, MD; Seung-Ho Hur, MD; Seung-Jae Joo, MD; Seung-Jea Tahk, MD; Seung-Jung Park, MD; Shung-Chull Chae, MD; Soo-Joong Kim, MD; Tae-Hoon Ahn, MD; Taek-Jong Hong, MD; Yang-Soo Jang, MD; YoungJo Kim, MD; Young-Keun Ahn, MD; Young-Youp Koh, MD; Wook-Sung Chung, MD. 